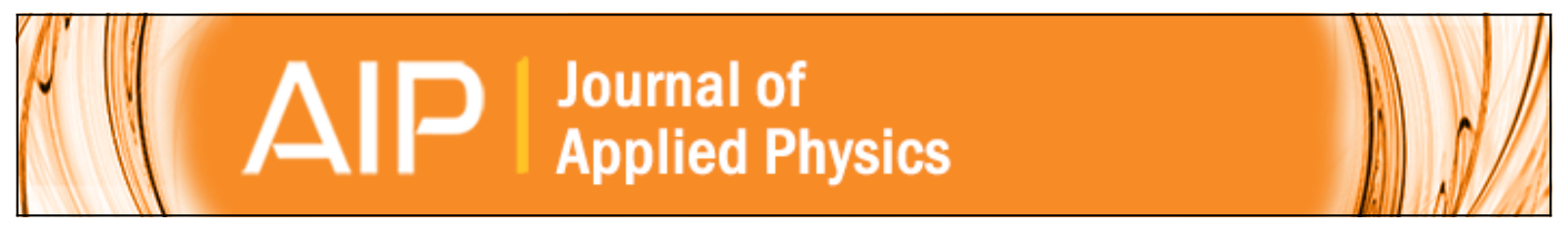

\title{
Structural and optoelectronical characterization of Si - SiO 2 / SiO 2 multilayers with applications in all Si tandem solar cells
}

D. Maestre, O. Palais, D. Barakel, M. Pasquinelli, C. Alfonso, F. Gourbilleau, M. De Laurentis, and A. Irace

Citation: Journal of Applied Physics 107, 064321 (2010); doi: 10.1063/1.3309761

View online: http://dx.doi.org/10.1063/1.3309761

View Table of Contents: http://scitation.aip.org/content/aip/journal/jap/107/6?ver=pdfcov

Published by the AIP Publishing

\section{Articles you may be interested in}

Charge photo-carrier transport from silicon nanocrystals embedded in SiO2-based multilayer structures

J. Appl. Phys. 112, 024324 (2012); 10.1063/1.4737579

Damage free laser ablation of $\mathrm{SiO} 2$ for local contact opening on silicon solar cells using an a-Si:H buffer layer J. Appl. Phys. 107, 043518 (2010); 10.1063/1.3309382

Silicon-rich $\mathrm{SiO} 2$ / SiO 2 multilayers: A promising material for the third generation of solar cell J. Appl. Phys. 106, 013501 (2009); 10.1063/1.3156730

Influence of the annealing treatments on the luminescence properties of $\mathrm{Si} \mathrm{O} / \mathrm{Si} \mathrm{O} 2$ multilayers J. Appl. Phys. 100, 123504 (2006); 10.1063/1.2402976

Influence of alloy composition on carrier transport and solar cell properties of hydrogenated microcrystalline silicon-germanium thin films

Appl. Phys. Lett. 89, 142115 (2006); 10.1063/1.2358318

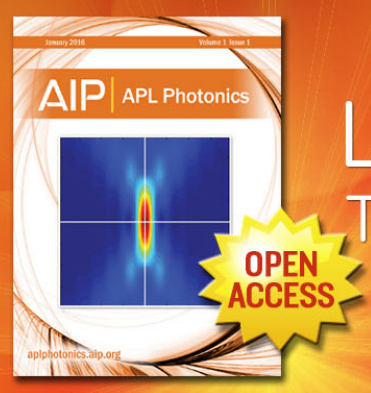

Launching in 2016! The future of applied photonics research is here 


\title{
Structural and optoelectronical characterization of $\mathrm{Si}-\mathrm{SiO}{ }_{2} / \mathrm{SiO}_{2}$ multilayers with applications in all Si tandem solar cells
}

\author{
D. Maestre, ${ }^{1}$ O. Palais, ${ }^{1, a)}$ D. Barakel, ${ }^{1}$ M. Pasquinelli, ${ }^{1}$ C. Alfonso, ${ }^{1, b)}$ F. Gourbilleau, ${ }^{2}$ \\ M. De Laurentis, ${ }^{3}$ and A. Irace ${ }^{3}$ \\ ${ }^{1}$ IM2NP, UMR CNRS 6242, Université Paul Cézanne-Aix-Marseille III, Av. Escadrille Normandie Niemen, \\ 13397 Marseille Cedex 20, France \\ ${ }^{2}$ CIMAP, UMR CEA/CNRS/Ensicaen/Université de Caen, 6 Boulevard Maréchal Juin, 14050 Caen Cedex 4, \\ France \\ ${ }^{3}$ Department of Biomedical, Electronic and Telecommunication Engineering, University of Naples Federico \\ II, Via Claudio 21, 80125 Naples, Italy
}

(Received 14 October 2009; accepted 11 January 2010; published online 31 March 2010)

\begin{abstract}
$\mathrm{SiO}_{2}$ multilayers with embedded $\mathrm{Si}$ nanocrystals ( $\mathrm{Si}$-ncs) were investigated as an approach for developing highly efficient all Si tandem solar cells. The nanostructured samples, fabricated by means of a reactive magnetron sputtering, were structurally and optoelectronically characterized using different techniques. High resolution transmission electron microscopy (TEM) and energy filtered images in TEM show a high density of Si-nc with uniform sizes below $4 \mathrm{~nm}$, while electrical characterization indicates high resistance values $\left(10^{2} \mathrm{k} \Omega\right)$ of these samples. In order to develop a better understanding of the optoelectronical behavior, photocurrent I-V curves were measured, obtaining variations under "dark" or "illumination" conditions. Recombination lifetimes in the order of tenths of nanoseconds were estimated by applying the transverse pump/probe technique. (C) 2010 American Institute of Physics. [doi:10.1063/1.3309761]
\end{abstract}

\section{INTRODUCTION}

Silicon nanostructures are considered to be among the most outstanding candidates to spread new applications in many interesting fields, such as optoelectronic, micro- and nanoelectronic researches. ${ }^{1,2}$ For instance, silicon nanostructures have attracted great interest in recent years in the photovoltaic field due to their promising electrical and optical properties as well as their capability to overcome some classical limitations, such as the bulk Si restricted conversion efficiency. The quantum effects associated with the size of the silicon nanoparticles, which permits bandgap tuning, increase the absorption and efficiency, or even enhance the carrier transport, as demonstrated by third generation photovoltaic cells. ${ }^{3,4}$ Indeed if $\mathrm{Si}$ nanoparticles are integrated in silicon based matrices, such as $\mathrm{SiO}_{2}, \mathrm{SiN}$, or $\mathrm{SiC}$, a completely silicon based cell could be developed, facilitating the improvement of integrated silicon technology. Some recent works $^{5-7}$ analyzed the morphology and optical properties of these structures, although few works were focused on electrical characterization. Recently different techniques have been used in order to fabricate silicon nanocrystals (Si-ncs), such as $\mathrm{Si}$ ion implantation, molecular beam epitaxy, laser ablation, and sputtering or plasma enhanced chemical vapor deposition. ${ }^{8-11}$ This work reports on the structural and optoelectronical characterizations of silicon-rich silicon oxide (SRSO) $/ \mathrm{SiO}_{2}$ multilayers (MLs) built by reactive magnetron sputtering. The structural characterizations have been carried out by means of high resolution transmission electron microscopy (HRTEM) in combination with electron energy loss

\footnotetext{
a) Author to whom correspondence should be addressed. Electronic mail: olivier.palais@univ-cezanne.fr.

${ }^{b)}$ Electronic mail: claude.alfonso@univ-cezanne.fr.
}

spectroscopy (EELS), while lifetime measurements and I/V curves under dark and illumination conditions were performed to analyze the optoelectronical properties. The correlation between the optoelectronical and the structural properties will contribute to the achievement of a deeper understanding of these systems as well as to their spread as future applications.

\section{EXPERIMENTS}

The samples analyzed in this work consist of a ML structure grown by reactive magnetron sputtering on a p-type (100)-oriented Si substrate. The ML growth process alternates the deposition of stoichiometric $\mathrm{SiO}_{2}$ followed by Sirich $\mathrm{SiO}_{2}$ (SRSO), as a function of the plasma (argon or hydrogen-rich, respectively) used during the sputtering of a pure $\mathrm{SiO}_{2}$ target at a $0.76 \mathrm{~W} \mathrm{~cm}{ }^{-2}$ power density. ${ }^{12}$ The argon and hydrogen partial pressures used during the growth are 1.5 and $6.0 \mathrm{~Pa}$, respectively. A subsequent annealing at $1100{ }^{\circ} \mathrm{C}$ results in segregation of $\mathrm{Si}$ and $\mathrm{SiO}_{2}$ within the SRSO layer and allows defects in the $\mathrm{SiO}_{2}$ matrix to repair. ${ }^{13,14}$ The analyzed MLs, named as M1 to M6, consist of different numbers and thicknesses of $\left(\mathrm{SRSO} / \mathrm{SiO}_{2}\right)$ bilayers as indicated in Table I. The thickness of each individual layer is controlled by the deposition time under $\mathrm{Ar}$ or $\mathrm{Ar}$ $+\mathrm{H}_{2}$ plasma. For samples M1-M2, the $\mathrm{SiO}_{2}$ layer thickness was held to $3.5 \mathrm{~nm}$, while for the rest of the samples, it was $1.5 \mathrm{~nm}$. The SRSO layer thickness varied from 1.5 to $8 \mathrm{~nm}$. Different specimens of M1 and M2 MLs were prepared either for plan-view or cross-sectional analysis in the TEM. For the former, conventional mechanical and $\mathrm{Ar}^{+}$ion polishing was used, while the latter were prepared by using the focused $\mathrm{Ga}^{+}$ion beam (FIB) milling process to achieve electron transparency. 
TABLE I. Number and thickness of $\left(\mathrm{SRSO} / \mathrm{SiO}_{2}\right)$ bilayers for each $\mathrm{ML}$ investigated (M1-M6).

\begin{tabular}{lcc}
\hline \hline Sample name & Number of bilayers & $\begin{array}{c}\text { Thickness of SRSO layer } \\
(\mathrm{nm})\end{array}$ \\
\hline M1 & 120 & 4 \\
M2 & 240 & 4 \\
M3 & 1.5 & 8 \\
M4 & 5.5 & 1.5 \\
M5 & 3.5 & 3 \\
M6 & 2.5 & 6 \\
\hline \hline
\end{tabular}

High resolution TEM as well as energy filtered images (EF-TEM) were performed in a TEM JEOL-2010 F operating at $200 \mathrm{kV}$ and equipped with a Gatan energy filter. EFTEM based on the plasmon peaks were recorded using a 5 $\mathrm{eV}$ slit in the energy selecting plane of the filter. This technique can also provide chemical information down to the nanometer scale. ${ }^{15}$ To estimate the sample recombination lifetime, which is a direct indicator of the capability on photogeneration, free carrier absorption measurements were performed using the transversal pump and probe technique. ${ }^{16}$ This method uses a continuous laser beam with a subbandgap energy (wavelength $\lambda=1550 \mathrm{~nm}$ ) as optical probe of the absorption coefficient variation induced by the excess of free carriers generated inside the sample by a laser pulse with energy greater than the sample bandgap (pump wavelength $\lambda<1100 \mathrm{~nm}$ ). The probe beam propagates along the sample thickness, parallel to the sample surface illuminated by the pump pulse. The transmitted beam through the sample detected by means of an ultrafast InGaAs photodiode gives immediate information about the sample recombination lifetime. After the excitation of the pump beam, the timedependent decay curve consists of an absorption peak followed by an exponential return to the unperturbed continuous level (steady state). The time-varying slope of this transient gives a direct measurement of the recombination lifetime. ${ }^{17,18}$

For the electrical measurements a Van der Pauw method using a four-probe configuration was employed. I/V curves were acquired at room temperature in dark or illuminated conditions by using a Keithley 4200 semiconductor characterization system. Photocurrent experiments were carried out using different wavelengths generated by light emitting diodes (LED's) emitting at 375 and $940 \mathrm{~nm}$.

\section{RESULTS AND DISCUSSION}

Figure 1(a) displays a HRTEM image of the crosssectional specimens in which the different piled layers which form the MLs can be distinguished. These layers present a good periodicity as well as an average thickness around 3.5 $\mathrm{nm}$. It is not possible, however, to observe the individual $\mathrm{Si}$ nanocrystals (Si-ncs) within the SRSO layer using HRTEM. This is due either to misorientation of the Si-ncs with respect to the e-beam or to amorphization generated during the FIB specimen preparation which requires the use of high energy $\mathrm{Ga}^{+}$ions. In order to overcome this limitation and to resolve the presence of Si nanoparticles in the dielectric matrix, an
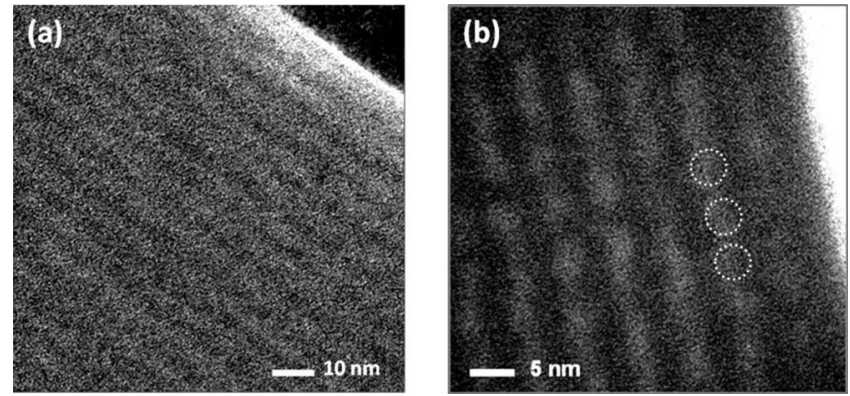

FIG. 1. (a) Cross-sectional HRTEM micrograph showing the multilayered structure corresponding to sample M1. (b) EF-TEM image of the same specimen where the Si nanoparticles can be identified as bright features.

imaging technique based on EELS was also employed. The areas of contrast in the EF images (EF-TEM) are directly related to absorption differences in the plasmon region (low energy) and are characteristic of the energy loss of electrons when interacting with the sample. ${ }^{19}$ Theoretical plasmon peaks of $\mathrm{Si}$ and $\mathrm{SiO}_{2}$ are centered at around 16 and $24 \mathrm{eV}$, respectively, permitting the identification of each element in the analyzed region of the specimens, even in the case of amorphous material. In this work, EF images were generated by recording each characteristic signal with an energy slit of $5 \mathrm{eV}$. It should be noted that EF-TEM images were obtained in different regions of the samples, leading us to conclude that the results presented in this work are representative of the studied structures. Figure 1(b) shows a typical EF image recorded at $16 \mathrm{eV}$ (Si plasmon peak) from sample M1. Similar results were obtained for the sample M2 which contains more stacked layers. In this EF-TEM image, a bright contrast is visible (on the right part) associated with the Si substrate as well as small round individual bright features. These white dots attest of the presence of Si nanoparticles (Si-nps) within the SRSO layers. The opposite contrast was observed in the $\mathrm{EF}$ images acquired at $24 \mathrm{eV}\left(\mathrm{SiO}_{2}\right.$ plasmon peak), not shown here, which confirms the correct identification of each phase by this technique. From the study of the EF-TEM images, the distribution, density, and size of the embedded Sinps can be analyzed. These Si-nps appear to be well distributed throughout the SRSO layers and present in a high concentration. The thickness of the SRSO layers and their confinement between two $\mathrm{SiO}_{2}$ layers permit to control the size of the Si-nps which is one of the advantages of the MLs structure. This effect results in a higher uniformity of Si-nc sizes ( $3.5 \mathrm{~nm}$ diameter on average), which is an important parameter for the future optoelectronic devices. The analysis of the filtered images must take into account that the observed Si-nps could be the result of the projection from different planes resulting from the preparation of the cross sections. That is why MLs were prepared in plan-view configuration to complete the microstructural study and to achieve a better estimation of the density and distribution of Si-nps into the layers. Figure 2 shows a HRTEM image corresponding to the M1 MLs in plan-view observation. Contrary to the cross-sectional images, such an approach allows the observation of individual Si-nps, as rounded shapes with an average diameter statistically estimated to be of $3.5 \mathrm{~nm}$. The concomitant analysis of both plan and cross-sectional 


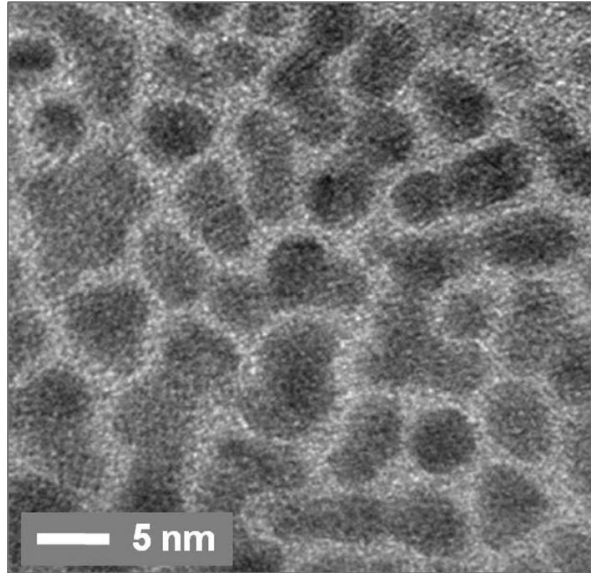

FIG. 2. Plan-view HRTEM micrograph of sample M1 showing Si nanoparticles embedded in the $\mathrm{SiO}_{2}$.

views leads us to conclude that these $\mathrm{Si}$ nanoparticles are spherical, as expected due to energy minimization. The Si-np density estimated from the HRTEM images is 4 $\times 10^{+12} \mathrm{~cm}^{-2}$ which when factored with MLs structure leads to a total density of $9 \times 10^{18} \mathrm{~cm}^{-3}$. Under these conditions the conductivity is high enough to warrant high efficiency solar cells. Indeed, according to the images Si-nps are separated from each other by about $1 \mathrm{~nm}$. This distance is favorable for the achievement of good electrical transport properties based on the tunneling probability in a $\mathrm{SiO}_{2}$ matrix, as referred by Conibeer et al. ${ }^{20}$ Furthermore, contrary to other works, ${ }^{21,22}$ no coalescence between Si-nc or percolation effects were observed, which attest of the quality of the fabricated MLs and the precise control of the excess Si permitted by the reactive process. The optoelectronical properties of these samples were investigated, in order to correlate them to the structural characteristics. The Van der Pauw "four point probes" technique was chosen in order to evaluate the $\mathrm{R} \square$ (square resistance). As shown in Fig. 3, both M1 and M2 MLs present high $\mathrm{R} \square$ values (235 and $302 \mathrm{k} \Omega$, respectively) at room temperature, basically due to the dielectric character of the matrix. However, these values are lower than those of pure $\mathrm{SiO}_{2}$, which could suggest the onset of conductivity effects arising from the presence of Si-nc. The resistivity at room temperature for each sample can be deduced from the $\mathrm{R} \square$ values by taking into account sample thickness (e)

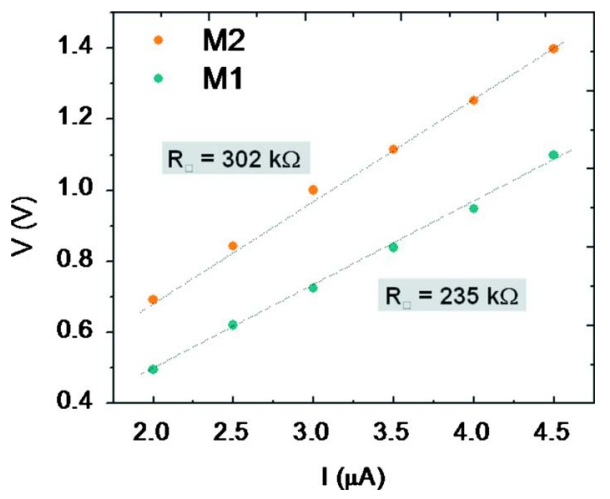

FIG. 3. (Color online) I-V curves obtained with the Van der Pauw technique on samples M1 and M2.

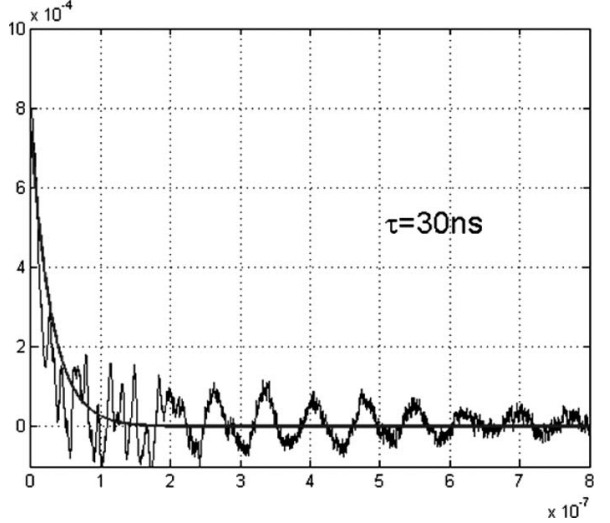

FIG. 4. Normalized signal of the pump and probe lifetime measurement on sample M2. The signal is acquired synchronized to the exciting pump pulse, so that it shows the transient after the pulse is turned off (pump pulse width about $7 \mathrm{~ns}$ ).

and the positions of the tips. Values of 77 and $197 \Omega . \mathrm{cm}$ were obtained for samples M1 and M2, respectively, similar to those referred to by Conibeer et al. ${ }^{3}$ These samples being similar except for the thickness, the difference of $\mathrm{R} \square$ values can therefore be explained either by a possible contribution from the Si substrate in the case of M1 MLs and/or by a higher density of structural defects due to higher number of interfaces for M2 MLs. In this latter case, such defects could affect negatively the carrier transport process and hence increase $\mathrm{R} \square$. In order to estimate the photogeneration ability of these MLs, pump and probe measurements were performed. The characteristics of our setup were such that only thicker M2 MLs were analyzed avoiding any possible contribution from the $\mathrm{Si}$ substrate in the measurement. The recorded data are reported in Fig. 4. A characteristic absorption peak can be observed once the pulse beam is turned off. The pulse wavelength is $\lambda=910 \mathrm{~nm}$ while its pulse width is about $7 \mathrm{~ns}$. The exponential constant is a direct measure of the sample lifetime and was estimated to be about $30 \mathrm{~ns}$ by fitting to the experimental data. Such a fast characteristic time cannot be ascribed to the silicon substrate, since its recombination constant is orders of magnitude greater (microseconds or even more). This means that the recombination processes measured within the ML are not influenced by the substrate. These effects will be taken into account when analyzing the photovoltaic behavior of this sample. Various I/V curves were recorded at room temperature on M2 MLs under dark or illumination conditions with two different wavelengths. The measurements were carried out using two microtips placed onto aluminum Ohmic contacts that have previously been deposited onto the sample. To improve contact quality and to allow the contacts to penetrate through the surface oxide into the layers, a $15 \mathrm{~h}$ bake at $500{ }^{\circ} \mathrm{C}$ in a N2 atmosphere was performed after the $\mathrm{Al}$ deposition. The nonuniform spiking of the annealed $\mathrm{Al}$ generates a large surface area of contact, thus achieving a small contact resistance. As observed in Fig. 5, the current voltage curves show a linear behavior. The initial results obtained when illuminating under "white light" [Fig. 5(a)] indicate a decrease ( 20\%) in the resistance from 48 to $37 \mathrm{k} \Omega$, which can be attributed to an increase in the charge carriers and hence confirming the 

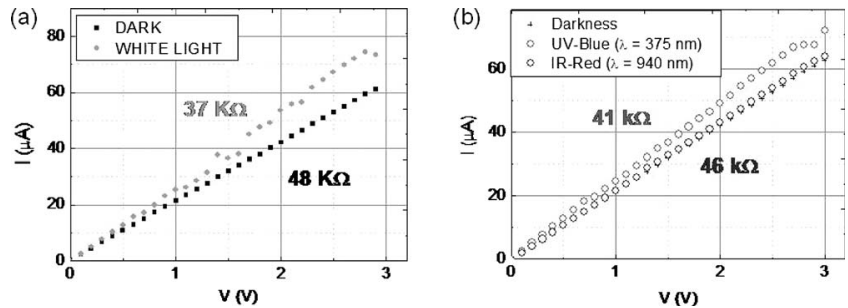

FIG. 5. I-V curves recorded on sample M2 under either illumination or dark conditions (a) or by using different $\lambda(b)$.

photovoltaic behavior of our MLs. In order to control the penetration depth and the photon energy, i.e., to separate the contributions of the substrate and of the Si-nps present in the SRSO layers, MLS were illuminated by different wavelengths. The use of UV-blue $(\lambda=375 \mathrm{~nm})$ permits the selective study of the surface area (including the Si-nc), while using the near IR-red $(\lambda=940 \mathrm{~nm})$ allows the analysis of a possible contribution of the Si substrate. Electron-hole pairs in the Si-nps could easily be generated when using UV-blue excitation, in view of the absorption cross section of Si-nps and the wavelengths. ${ }^{23} \mathrm{As} \mathrm{SiO}_{2}$ acts as a dielectric, no contribution from the matrix is expected. After illumination under IR-red light, the I/V curve remains similar to the one corresponding to the dark conditions [as shown in Fig. 5(b)]. Only a negligible decrease of about $3 \%$ of the resistance is observed. These results confirm that the contribution from the substrate to the measured conductance can be neglected. Therefore, the main electrical conduction path is a consequence of the existence of Si-nps within the MLs. When illuminating under UV-blue light, the I/V curve [Fig. 5(b)] presents a similar behavior to that obtained under white light with a corresponding resistance value of $41 \mathrm{k} \Omega$. Using this experimental approach, we can assume that the photogenerated carriers are responsible of the improvement of the conductivity. The resistance values of our Mls are lower than those reported recently by other authors such as Rölver et $a l{ }^{24}$ This difference can be attributed to the higher crystallinity and the optimized spacing between Si-nps. The size and density of Si-nps in our MLs demonstrate the presence of small Si-nps interspacing (1-2 nm) which favors conduction mechanisms through the tunnel effect. Green et al. ${ }^{25}$ stated that Si-nc should be separated less than $2 \mathrm{~nm}$ in order to exhibit photovoltaic effects, in agreement with the Si-np distribution observed in our layers. The $\mathrm{R}(\Omega)$ deduced from I/V curves performed under UV light on thinner MLs (M3M6) are reported in Fig. 6. The result analyze demonstrates that the photovoltaic effect is dependant on the Si-np size. Reducing the Si-nps allows an increase in conductivity under illumination. For Si-nps with a diameter greater than $5 \mathrm{~nm}$, no photocurrent effect is observed which is attributed to the loss of quantum efficiency in Si-nps. As observed previously for the M1 and M2 MLs, I/V characteristics are not modified by IR illumination. This result is a good proof that the optoelectronic and photovoltaic behavior of Si-nc arises as a consequence of its gap value close to $1.7 \mathrm{eV}$. A photovoltaic effect is clearly observed.

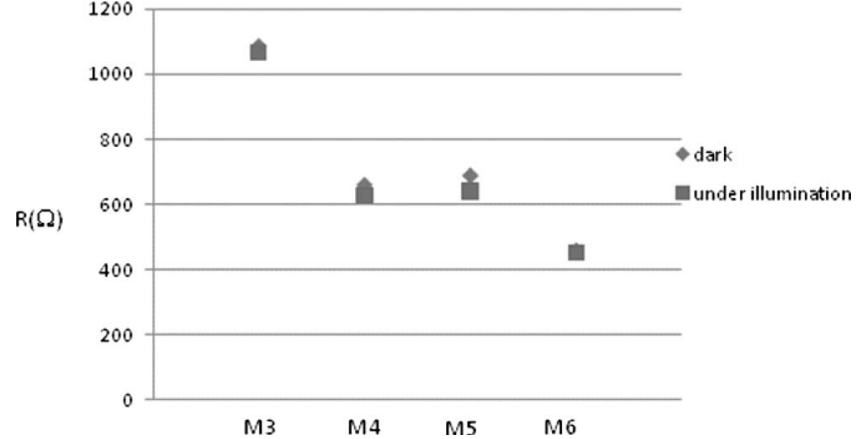

FIG. 6. $\mathrm{R}(\Omega)$ acquired on samples M3-M6 under illumination or dark conditions.

\section{CONCLUSIONS}

The structural and optoelectronical properties of $\mathrm{Si}$ nanoparticles in Si-rich $\mathrm{SiO}_{2} / \mathrm{SiO}_{2}$ MLs fabricated by reactive magnetron sputtering have been analyzed. The size and uniformity of these nanoparticles are well controlled by the layer's thickness and the reactive process as evidenced by HRTEM and EF-TEM images. The microstructural analysis has demonstrated the spherical shape of the Si nanoparticles as well as their high density with value close to $10^{+19} \mathrm{np} \mathrm{cm}^{-3}$ reached. The electrical experiments performed on the MLs have shown a low recombination lifetime value of about $30 \mathrm{~ns}$ and high resistivity of about $102 \mathrm{k} \Omega$. Photovoltaic effect depending on the Si-np size has been observed when MLs are illuminated with different monochromatic blue and UV light sources (wavelength: 405 and $375 \mathrm{~nm}$ ). This is a clear demonstration that quantum confinement of the carrier plays a role in the photocourant process. Such a result seems suitable for the implementation of $\mathrm{Si}$ nanoparticles in all Si tandem solar cells.

\section{ACKNOWLEDGMENTS}

French authors thank the ANR Solaire Photovoltaïque (National Research Agency) for financial support of this work through the project DUOSIL. We thank also C. Dominici and W. Saikaly from CP2M for their contribution to HTREM observations.

A.I. thanks the MIUR for financial support under the FIRB framework

${ }^{1}$ M. A. Green, J. H. Zhao, A. H. Wang, P. J. Reece, and M. Gal, Nature (London) 412, 805 (2001).

${ }^{2}$ M. A. Makeev, J. Nanoelectron. Optoelectron. 1, 176 (2006).

${ }^{3}$ G. Conibeer, M. Green, R. Corkish, Y. Cho, E.-C. Cho, C.-W. Jiang, T. Fangsuwannarak, E. Pink, Y. Huang, T. Puzzer, T. Trupke, B. Richards, A. Shalav, and K.-L. Lin, Thin Solid Films 511-512, 654 (2006).

${ }^{4}$ W. Hoffmann, Sol. Energy Mater. Sol. Cells 90, 3285 (2006).

${ }^{5}$ M. Zacharias, J. Heitmann, R. Scholtz, U. Kahler, M. Schmidt, and J. Bläsing, Appl. Phys. Lett. 80, 661 (2002).

${ }^{6}$ T.-W. Kim, C.-H. Cho, B.-H. Kim, and S.-J. Park, Appl. Phys. Lett. 88, 12302 (2006).

${ }^{7}$ J. Y. Fan, X. L. Wu, and P. K. Chu, Prog. Mater. Sci. 51, 983 (2006).

${ }^{8}$ N. P. Stepina, A. V. Dvurechenskii, V. A. Ambrister, J. V. Smagina, V. A. Volodin, A. V. Nenashev, J. P. Leitao, M. C. do Carmo, and N. A. Sobolev., Thin Solid Films 517, 309 (2008).

${ }^{9}$ S. K. Yang, W. P. Cai, H. B. Zeng, and Z. G. Li, J. Appl. Phys. 104, 023516 (2008).

${ }^{10}$ C. Ternon, F. Gourbilleau, X. Portier, P. Voivenel, and C. Dufour, Thin Solid Films 419, 5 (2002). 
${ }^{11}$ B.-H. Kim, C.-H. Cho, T.-W. Kim, N.-M. Park, G.-Y. Sung, and S.-J. Park, Appl. Phys. Lett. 86, 091908 (2005).

${ }^{12}$ C. Ternon, C. Dufour, F. Gourbilleau, and R. Rizk, Eur. Phys. J. B 41, 325 (2004).

${ }^{13}$ E. Talbot, R. Lardé, F. Gourbilleau, C. Dufour, and P. Pareige, EPL 87, 26004 (2009).

${ }^{14}$ F. Gourbilleau, X. Portier, C. Ternon, P. Voivenel, R. Madelon, and R. Rizk, Appl. Phys. Lett. 78, 3058 (2001).

${ }^{15}$ W. Grogger, B. Schaffer, K. Krishnana, and F. Hofer, Ultramicroscopy 96, 481 (2003).

${ }^{16}$ Z. G. Ling, P. K. Ajmera, M. Anselment, and L. F. Di Mauro, Appl. Phys. Lett. 51, 1445 (1987).

${ }^{17}$ L. Sirleto, A. Irace, G. F. Vitale, L. Zeni, and A. Cutolo, J. Appl. Phys. 93, 3407 (2003).

${ }^{18}$ A. Irace, F. Sorrentino, and G. F. Vitale, Sol. Energy Mater. Sol. Cells 84, 83 (2004).

${ }^{19}$ S. Schamm, C. Bonafos, H. Coffin, N. Cherkashin, M. Carrada, G. Ben Assayag, A. Claverie, M. Tencé, and C. Colliex, Ultramicroscopy 108,
346 (2008).

${ }^{20}$ G. Conibeer, M. Green, E.-C. Cho, D. König, Y.-H. Cho, T. Fangsuwannarak, G. Scardera, E. Pink, Y. Huang, T. Puzzer, S. Huang, D. Song, C. Flynn, S. Park, X. Hao, and D. Mansfield, Thin Solid Films 516, 6748 (2008).

${ }^{21}$ Y. Q. Wang, R. Smirani, G. G. Ross, and F. Schiettekatte, Phys. Rev. B 71, 161310 (2005).

${ }^{22}$ M. Carrada, A. Zerga, M. Amann, J. J. Grob, J. P. Stoquert, A. Slaoui, C. Bonafos, and S. Schamm, Mater. Sci. Eng., B 147, 218 (2008).

${ }^{23}$ D. Kovalev, J. Diener, H. Heckler, G. Polisski, N. Künzner, and F. Koch, Phys. Rev. B 61, 4485 (2000).

${ }^{24}$ R. Rölver, B. Berghoff, D. L. Bätzner, B. Spangenberg, and H. Kurz, Appl. Phys. Lett. 92, 212108 (2008).

${ }^{25}$ M. A. Green, E.-C. Cho, Y. Cho, Y. Huang, E. Pink, T. Trupke, A. Lin, T. Fangsuwannarak, T. Puzzer, G. Conibeer, and R. Corkish. Proceedings of the 20th European Photovoltaic Solar Energy Conference, Barcelona, June 2005 (unpublished), p. 3 